\section{La investigación científica universitaria y su relación con el desarrollo empresarial en el Perú}

University scientific research and its relationship with business development in Perú

\section{José Adolfo Hinojosa Pérez | Fernando Polo Orellana}

\section{RESUMEN}

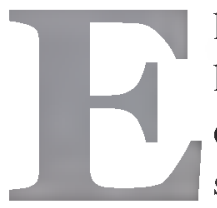

1 objetivo de este trabajo de investigación es el de estudiar la relación que existe entre la investigación científica universitaria, y el desarrollo del sector empresarial en el Perú. Así, en el estudio, se hace en primer lugar un análisis del rol del Estado en la ejecución de sus políticas educativas, y en ese contexto, luego se hace un diagnóstico situacional de la universidad peruana en la actualidad, centrando el interés en el estado y el desarrollo de la investigación e innovación científica en esta. Para después, examinar la existencia de vínculos y relaciones científico-tecnológicas con el sector empresarial del país. El método de investigación, que se utiliza para el desarrollo de este trabajo es de tipo exploratorio, descriptivo y analítico, entre otros. Los resultados de la investigación, confirman y comprueban la hipótesis y objetivo propuestos; es decir, que la universidad peruana, es un ente, que no genera investigación científica, o si lo hace, sus aportes son mínimos. Por tanto, no crea conocimiento nuevo y relevante, que sea provechoso para la propia universidad y para la sociedad, en este caso en particular, para el sector privado productivo del país. Lo que demuestra que la investigación científica universitaria no se relaciona favorablemente con el desarrollo empresarial en el Perú. Constituyéndose, por tanto, en una limitante para el impulso del crecimiento y desarrollo integral del país.

Palabras clave: universidad; investigación científica; empresa; desarrollo económico.

\section{ABSTRACT}

The objective of this research work is to study the relationship that exists between university scientific research and the development of the
José Adolfo Hinojosa Pérez jhinojosap@unmsm.edu.pe

Facultad de Ciencias Económicas

Universidad Nacional Mayor San Marcos

Lima, PERÚ

Fernando Polo Orellana d.fpolo@upla.edu.pe

Universidad Peruana de Los Andes Huancayo, PERÚ

COMO CITAR ESTE ARTÍCULO Hinojosa, Pérez J. A., Polo Orellana, F. (2021). La investigación científica universitaria y su relación con el desarrollo empresarial en el Perú. Revista de la Facultad de Ciencias Económicas, 27 (2), 117- 133 .

http://dx.doi.org/10.30972/rfee.2725661

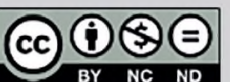

https: //creativecommons. org/licenses/by-nc-nd/4.0/ Revista de la Facultad de Ciencias Económicas ISSN 1668-6357 (formato impreso) ISSN 1668-6365 (formato digital) por Facultad de Ciencias Económicas Universidad Nacional del Nordeste (UNNE) Argentina se distribuye bajo una Licencia Creative Commons Atribución - No Comercial - Sin Obra Derivada 4.o Internacional. 
business sector in Peru.Thus, in the study, first an analysis of the role of the State in the execution of its educational policies is made, and in this context, then a situational diagnosis of the Peruvian university at present is made, focusing the interest on the state. and the development of scientific research and innovation in it. To later examine the existence of scientifictechnological links and relationships with the country's business sector. The research method used for the development of this work is exploratory, descriptive and analytical, among others. The results of the investigation confirm and verify the proposed hypothesis and objective; In other words, the Peruvian university is an entity that does not generate scientific research, or if it does, its contributions are minimal. Therefore, it does not create new and relevant knowledge that is beneficial for the university itself and for society, in this particular case, for the productive private sector of the country. This shows that university scientific research is not favorably related to business development in Peru. Therefore, becoming a limitation for the promotion of growth and integral development of the country.

Keywords: university; scientific research; company; economic development.

\subsection{INTRODUCCIÓN}

Según Larraín y Sachs (2004), haciendo alusión al famoso residuo de Solow, confirman que los EEUU “crecieron más por inspiración que por transpiración", a diferencia de la gran mayoría de países del mundo, como el caso del Perú y en general en los países latinoamericanos. Pues la evidencia sugiere que la causa del crecimiento económico del país, obedecería principalmente a mas por "transpiración que en inspiración", que explicaría, los limitados niveles de desarrollo del país. Esto por las razones siguientes: En primer lugar, la ausencia de innovaciones científicotecnológicas en los procesos productivos de las empresas; luego la falta de colaboración y cooperación entre la academia y el sector empresarial. Y la última, la insuficiencia en la asignación de recursos económicos a programas de investigación y desarrollo científico por parte del Estado y la propia empresa privada.

Por lo cual, en este trabajo de investigación nos proponemos estudiar las relaciones y los vínculos entre la academia y la empresa privada, como condición sine qua non, para alcanzar el desarrollo económico y social del país. En ese sentido, planteamos como problema general: ¿En qué medida la investigación científica universitaria se relaciona con el desarrollo empresarial en el Perú? Por la misma razón, nuestra hipótesis general es de: Demostrar que la investigación científica universitaria no se relaciona favorablemente con el desarrollo empresarial en el Perú. 


\subsection{REVISIÓN BIBLIOGRÁFICA (MARCO TEÓRICO)}

El año 2005, en el Perú, fue creado el Consejo Nacional de Ciencia, Tecnología e Innovación Tecnológica (CONCYTEC), cuya finalidad es la de normar, dirigir, fomentar, coordinar, supervisar y evaluar las acciones del Estado en el ámbito de la Ciencia, Tecnología e Innovación Tecnológica, así como promover e impulsar su desarrollo nacional mediante la acción concertada con otros organismos públicos y privados. Este organismo está regido por la Ley Marco de Ciencia y Tecnología $\mathrm{N}^{\circ}$ 28303. Y para lograr la ejecución de este tipo de políticas, el CONCYTEC, creó el Sistema Nacional de Ciencia y Tecnología e Innovación Tecnológica (SINACYT), quien es el organismo encargado de articular a las instituciones públicas, a la Academia (Universidades públicas y privadas), al sector empresarial, a las organizaciones sociales y a todas las personas relacionadas con la investigación científica. (CONCYTEC, 2020).

El año 2016, el gobierno emitió el Decreto Supremo 015-2016-PCM, que aprueba la Política Nacional para el Desarrollo de la Ciencia, Tecnología e Innovación Tecnológica (CTI), relanzando las politicas de impulso a las actividades y labores de investigación científica y tecnológica en el país.

De esta forma, en los últimos años, el CONCYTEC (2017) viene promoviendo acciones destinadas a impulsar el desarrollo de la investigación científica y tecnológica en el país. Tal es el caso de la puesta en marcha del "Plan de Actividades y Convocatorias 2018-2021 del Proyecto de Mejoramiento y Ampliación de los Servicios del Sistema Nacional de Ciencia, Tecnología e Innovación Tecnológica (Sinacyt)", el mismo que contempla una inversión total de US \$ 100 millones de dólares, de los cuales US \$ 55 millones corresponden a recursos estatales y US \$ 45 millones a un préstamo del Banco Mundial (BM).

Estos recursos estan orientados a desarrollar proyectos en seis sectores claves para el desarrollo económico y social del país: Agroindustria (alimentos procesados, pesca y acuicultura); Forestal; Textil y Confecciones; Minería; Manufactura avanzada, así como Ecoturismo, Restauración e Industrias Creativas (CONCYTEC, 2018). Igualmente, existe otro convenio de financiamiento firmado el 2018, entre este organismo y el BM, orientado a vincular y promover iniciativas para asociar la academia con la industria, el mismo que asciende a S/ 11.5 millones de soles.

Existen también otros proyectos, tal como los Proyectos de Aceleración de la Innovación, tambien con recursos del CONCYTEC, que buscan activar el despegue comercial de la innovación del producto (bien o servicio), proceso o modelo de negocio, implementados por empresas o emprendimientos locales preferentemente a partir de actividades de investigación y desarro1lo, privilegiando el desarrollo de la innovación en varias áreas del conocimiento (FONDECYT, 2019). Asi mismo, el Ministerio de la Producción, viene desarrollando en los ùltimos años, el Programa Nacional de Innovación para la Competividad y Productividad (Innovate Perú), que busca cofinanciar proyectos de innovación empresarial, desarrollo productivo, emprendimiento e instituciones del econsistema, a traves de concursos nacionales (Innovate Perú, 2020). 
Empero, los esfuerzos realizados en los últimos años por el Estado, para fomentar la innovacion y la investigación científica, son aún largamente insuficientes en términos de asignacion de recursos financieros, organización, infraestructura (laboratorios equipados), cooperación internacional. Puesto que el Perú, es uno de los países que menos gasta a nivel regional, en investigación y desarrollo, pues, según el Primer Censo Nacional de Investigación y Desarrollo (I+D) encargado el año 2016, por el CONCYTEC al Instituto Nacional de Estadística e Informática (INEI); nuestro país, destina para la investigación científica, tan solo el $0.08 \%$, del total de su PBI, mientras que otros países de la región como Colombia, gastaban el $0.25 \%$, en Chile se gasta el $0.38 \%$ y en México el $0.54 \%$ (Ver la Figura No 1). Este mismo censo, revela que, en el Perú, existe solo un investigador por cada 5.000 personas de la Población Económicamente Activa (PEA), en tanto que en países como Chile, tienen por cada mil 1.4, Brasil 2.5 investigadores y a nivel de la OCDE por cada mil, 3 investigadores (CONCYTEC, 2017). Según este mismo organismo, del total de investigadores, el $31.8 \%$ poseían el grado de doctor, el $34.3 \%$ tienen el grado de magister y el $26 \%$ poseía solo título profesional. En relación a los gastos en $\mathrm{I}+\mathrm{D}$ por área del conocimiento, según la Figura $\mathrm{N}^{\circ}$ 2, en el año 2015, el 32\% fue destinado a las ciencias naturales, el $22.7 \%$ al área de ingeniería y tecnología, el 19.3\% a ciencias sociales. En cuanto a la producción de patentes, por ejemplo, la Universidad Nacional Mayor de San Marcos, en el campo de las ciencias e ingeniería por parte de los investigadores, profesores y estudiantes, había solicitado en el periodo 2009-2013, solo dos patentes, y durante los años 2011-2012, la UNMSM, presenta 11 solicitudes de patentes, esto en el marco del Programa de Identificación de Materia Patentable llevado a cabo por INDECOPI (Chuquispuma, 2016).

Figura $\mathrm{N}^{0} 1$.

Gasto en I+D como Porcentaje del PBI

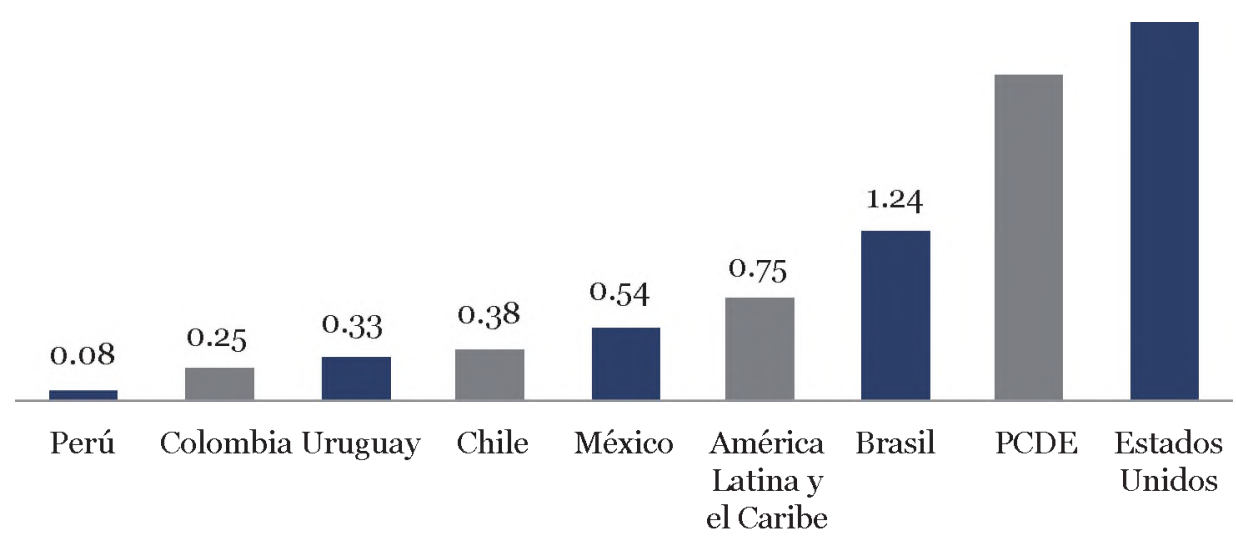

Fuente: 1 Censo Nacional de Investigación y Desarrollo. CONCYTEC.

Cifras del 2014, excepto para México (2013) y Perú (2015). 
Lo arriba expuesto concuerda con lo presupuestado para el sector educativo nacional, pues en el año 2018, los montos asignados, fueron tan solo del orden del 3.7\% del Producto Bruto Interno (PBI), lejos de un mínimo exigido (6\%) por diferentes estamentos de la sociedad peruana; tomando en consideración además que al 2011 el $75 \%$ de centros educativos (primaria y secundaria) eran públicos y el 25\% privados (Andina, 2011); y del total de universidades en el país, al 2019, 36\% eran públicas y el $64 \%$ privadas (SUNEDU, 2019). Lo cual revelaría las restricciones de parte del Estado en terminos de la provision de bienes publicos y el no reconocimiento de la importancia que tiene la educación y la investigación científica en el desarrollo del país. En ese sentido, la fracción asignada a la educación en el Perú, comparada con otros países de la región, es evidentemente mucho menor, pues Chile destinaba el 5.4\% de su PBI el 2017, Colombia el 2018, el 4.5\%, Argentina el 2017 el 5.5\% y Finlandia en Europa para el 2016 el 6.9\% (Banco Mundial, 2019).

Por ello, considerando que la investigación científica-tecnológica, es un factor crítico de éxito en un país, el Estado peruano, ha carecido durante décadas de políticas públicas, orientadas al desarrollo de la ciencia y tecnología. Lo que significa que ni la educación, ni el impulso a las actividades de investigación, significaron para este, una prioridad o preferencia nacional. No es, sino que, en los últimos años, el Estado a través del CONCYTEC viene impulsando una Política Nacional para el Desarrollo de la Ciencia, Tecnología e Innovación (CTI), arriba mencionada, movilizando algunos recursos financieros importantes y procurando con ello y de manera insuficiente aún, recuperar en parte, largos años perdidos en el avance de la ciencia y la tecnología en el país.

Figura $\mathrm{N}^{\circ} 2$.

Gasto en I+D por Area de Conocimiento, 2014-2016 (Porcentaje)

2015

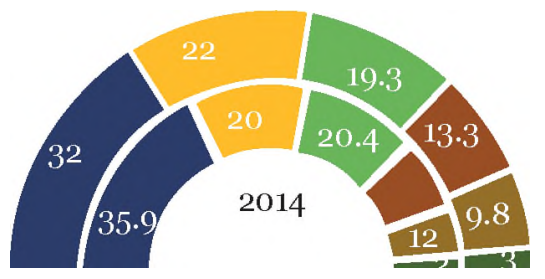

- Ciencias Naturales

* Ingenieria y Tecnologia

- Ciencias Sociales

- Ciencias Agrícolas

Fuente: I Censo Nacional de Investigacion y Desarrollo. CONCYTEC, 2016.

Entonces, al analizar el funcionamiento de las universidades públicas, comprobamos que el Estado peruano, no dota de los recursos financieros suficientes y necesarios para estas; con los cuales puedan cumplir con sus objetivos institucionales, tanto de formación profesional como de investigación científica. El año 2020, el presupuesto asignado para las universidades públicas es el 
14.6\% del total presupuestado para el sector educación, y representa aproximadamente el $0.6 \%$ del total del PBI (Perú21, 2020). Porque si en los hechos, las asignaciones presupuestales para cubrir los gastos de profesionalización de los estudiantes son insuficientes, el Estado provee mucho menos recursos aún para el impulso de la investigación científica y tecnológica a estos centros superiores de estudios. La consecuencia es, por tanto, que las universidades públicas peruanas, tengan una producción académica y científica limitada e invisible. Así, ninguna universidad pública supera las 500 publicaciones científicas en cinco años (Palmieri, 2017). Y en el plano internacional, las publicaciones de las universidades públicas en revistas indexadas es reducida, pues solo el 10.3\% tenía un impacto de ese tipo (CONCYTEC, 2017).

Por su parte las universidades privadas con y sin fines de lucro, si bien muestran mejores estadísticas en cuanto a ratios de investigación científica, los números no son sustancialmente diferentes. Así, el año 2015, del total de artículos científicos publicados en revistas indexadas de carácter nacional, el $47 \%$ correspondió a las universidades privadas sin y con fines de lucro, y el 34\% a las universidades del Estado (CONCYTEC, 2017). Así mismo, al año 2016, en el Perú, existían 3,374 investigadores, de los cuales 2,192 es decir el 65\% eran docentes universitarios, lo que significa el 2.6\% del total de profesores universitarios (Pulido, 2019).

En esa línea, es importante remarcar que ni la universidad privada ni la pública, desarrollan sus investigaciones y producción científica de forma regular y organizada; salvo algunas de las universidades más reconocidas. Este comportamiento demostraría otra de las principales fragilidades de las universidades en el Perú, pues, el limitado desarrollo de la investigación científica, no se realiza de manera integrada, sistematizada, estructurada y sostenible en el tiempo; sino de manera espontánea, circunstancial y eventual, es decir informal.

En cuanto a la relación entre la academia y la empresa, en el campo de la investigación y desarrollo científico, en países como el Perú; no solamente existen relaciones débiles, entre ellas; sino que tradicionalmente existe un divorcio entre ambas. Puesto que ni las universidades ni las empresas, han intentado desarrollar lazos, que les permita intercambiar conocimientos y recursos. Lejos de ello, cada una de estas instituciones, se desenvuelve en un mundo distinto y receloso, con espacios excluyentes entre unas y otras, sin contemplar la necesidad de la implementación y complementación de objetivos y estrategias de interés mutuo, ni tampoco vislumbrar el interés y los objetivos de desarrollo nacionales. Lo cual se explica por ejemplo en la desactualización e incompatibilidades de los contenidos curriculares básicos, y la inadecuación de su oferta profesional a los requerimientos actuales de las empresas y las necesidades estratégicas del país (Comisión Nacional por la Segunda Reforma Universitaria, 2002) ${ }^{1}$. Es así entonces, que la universidad no respondería a las exigencias de la empresa, en términos de la formación de profesionales que requieren estas últimas. Esto se revela, en el hecho que, en el Perú, se observa una sobrepoblación de estudiantes en ciencias sociales, y un déficit de profesionales en ciencias básicas e ingenierías. Estas últimas consideradas esenciales para la

${ }^{1}$ No obstante estar en vigencia la Reforma Universitaria, desde el 2014, los problemas señalados, no han cambiado de manera sustantiva. 
innovación y la dinámica de los procesos productivos de las propias empresas, sean estas grandes corporaciones, PYME o MYPE² ${ }^{2}$.

Pero el aspecto más relevante que explica el problema del desarrollo de la educación superior universitaria en el Perú, y su relación con la empresa privada, es que, en las universidades existe una preponderancia de un sistema educativo orientado principalmente a la profesionalización de los estudiantes, en perjuicio del desarrollo de una formación orientada a la vez al impulso de la investigación científica y tecnológica. En el caso de las universidades privadas, este problema es más agudo; pues gran parte de estas, responden a un criterio eminentemente mercantilista, dejando de lado criterios mínimos de calidad y eficiencia. Lo cual nos lleva a la conclusión de que estas descuidan no solamente sus funciones de formación académica, dirigida hacia la profesionalización de los estudiantes, sino que abandonan por completo el aliento a labores de investigación científica y tecnológica. Lo que origina finalmente repercusiones negativas en el sistema de la educación universitaria, en la educación nacional y en el desarrollo económico y social del país. ${ }^{3}$

Pero, cuáles son los tipos de vinculación que deben desarrollar las universidades y las empresas? Según Sarabia (2016), existen cuatro canales de relación entre ambas:

a) Información que incluye publicaciones, asistencia a conferencias con la participación de la empresa y universidad, creación de redes, asesoramiento o apoyo tecnológico.

b) Formación de recursos humanos, que incluye asesoramiento o apoyo tecnológico, contratación de posgraduados, entrenamiento de posgraduados en la industria, entrenamiento a empleados de la empresa, intercambio temporal de personal (a corto y largo plazo).

c) Servicios y productos de investigación, que incluye consultoría con investigadores individuales, investigación por contrato, investigación conjunta, cesión o explotación de patentes.

d) Instauración de empresas, que incluye incubadoras tecnológicas, parques científicos y tecnológicos (2016, p. 16).

La experiencia muestra que muy pocos o solo algunos canales de vinculación se han puesto en práctica, en el caso peruano; dentro de los cuales destacarían: el canal a) relativo a la asistencia a conferencias con la participación de la empresa, b) la formación de los profesionales en sus di-

\footnotetext{
${ }^{2}$ La tradición republicana, no ha cambiado mucho en relación al tipo de formación profesional colonial, donde en esta última, es sabido que, de cada una de las familias de la época, uno debería ser obligatoriamente abogado, el segundo sacerdote y el tercero militar. ${ }^{3}$ En los últimos años (Después del 2014), gracias a la nueva Ley Universitaria $N^{\circ} 30220$, se ha establecido un sistema de licenciamiento a las universidades tanto públicas como privadas y como consecuencia de ello, varias universidades sobre todo de carácter privado, han sido cerradas.
} 
ferentes especialidades (que es una función inherente a la universidad), luego la contratación de graduados y posgraduados, como parte del personal laboral de las empresas. Pero el que destaca por su intrascendencia en estos tipos de vínculos es el punto c) es decir, la falta de programas de investigación científica y tecnológica conjunta, para que luego esta sea transferida a las empresas en la forma de la cesión o explotación de patentes, en la forma de innovación tecnológica, entre otros. Por lo cual, consideramos que este es el punto frágil y endeble, que debe de revertirse, para convertirse en una relación fructuosa y productiva para la academia y la empresa.

¿Por qué entonces, este vínculo sería el más débil de entre todos aquellos mencionados? Una de las respuestas posibles como se ha ya adelantado, es que la universidad hace muy poca investigación o simplemente no la hace. Al respecto, Ismodes (2015), sostiene que las universidades en el Perú, comparadas con las de los países avanzados, no tienen mucho que ofrecer a las empresas. Puesto que en las universidades peruanas no existe conocimiento propio que se genere, ni menos existe capacidad para generarlo. Por lo cual, sin una oferta de actividades que interesen a las empresas en el campo del conocimiento, la vinculación no tiene mayor sentido.

No obstante, el problema de la transferencia del conocimiento científico, no solo es responsabilidad de las universidades, sino que es también de la empresa, quien en la práctica, dificulta que el conocimiento científico que podría ser generado en las universidades, sea incorporado en sus actividades; pues el tipo de especialización de la producción desarrollado en el Perú y general en América Latina, orientado hacia actividades de bajo contenido tecnológico, obstaculiza la dinámica innovadora, esto entonces, debido a que gran parte de los bienes de capital, los equipos de producción y los insumos (imputs) que utilizan las empresas nacionales, no son elaborados en los propios centros de producción locales, sino que se manufacturan en países que tienen en gran parte el monopolio en la generación y producción científica tecnológica en el mundo (Vega, 2011). La evidencia es que más de las dos terceras partes del total de las importaciones del país, es decir el $76.8 \%$, se componen de estos factores señalados, los cuales están dirigidos a satisfacer la demanda destinada a desarrollar los procesos de producción de las empresas (Banco Central de Reserva del Perú, 2019)4. Igualmente, otro obstáculo para una relación fructuosa entre la academia y la empresa, se ubica en las micro y pequeñas empresas, al desempeñarse estas últimas en sectores de poca intensidad tecnológica. Por lo cual, estas carecen de capacidades para poder absorber el conocimiento científico universitario (Vega, 2011), lo que finalmente no contribuye al desarrollo de intereses comunes en el campo de la investigación científica entre la universidad y el sector privado.

En ese contexto, las empresas en el Perú, no hacen investigación para el desarrollo (I+D) y si la hacen es mínima, pues ni las grandes corporaciones peruanas con recursos financieros considerables, ni mucho menos las PYME y MYPE la desarrollan. Según el BBVA (2014), citando a la Encuesta Nacional de Innovación en la Industria Manufacturera del año 2012; las empresas en el Perú, habían desarrollado actividades de “innovación”, en la compra de bienes de capital en el $78.9 \%$, que representa el mayor porcentaje, seguida por las actividades de capacitación con el

${ }^{4}$ Este tipo de dinámica en el comercio exterior, además genera un drenaje importante de divisas. 
48.7\%. Lo que significa una vez más que las empresas peruanas, en gran parte dedican recursos, para la importación de estos bienes que no son producidos en el país, sino en el extranjero. Es por ello, que esta misma institución, sostiene que la industria nacional, requiere mayores niveles de innovación que no solo abarquen inversiones en bienes de capital, sino que también inviertan en investigación y desarrollo, diseño de ingeniería industrial y capacitaciones (BBVA Research, 2014). En ese sentido, un indicador de que los emprendedores y las empresas privadas peruanas desarrollan una escasa actividad innovadora, es el número de solicitudes de patente por cada millón de habitantes, por ejemplo al 2019, según el WEF, esta cifra era de solo 0.27, comparado con Korea del Sur, que llega a 461.15, igualmente por cada millón de habitantes, y el Perú, ocupa el puesto 85 entre 141 países (World Economic Forum, 2019).

La consecuencia de lo expuesto, se expresa en los bajos niveles de innovación y competitividad del país, en relación al resto del mundo. Pues, de acuerdo al ranking de innovación global, elaborado por el Global Innovation Index (2019), el Perú, al año 2019, se sitúa en el puesto 69 de un total de 129 naciones, en tanto que Chile se ubica en el puesto 51 (siendo el primero de la región) y muy lejos del primer lugar que es Suiza. Adicionalmente, el informe de este organismo indica que el Perú muestra dos puntos débiles, por citar algunos. El primero, la divergencia en la colaboración en investigación entre universidad-empresa y el segundo, la ausencia de alianzas estratégicas para desarrollar acuerdos de joint venture relativos a la sofisticación de negocios. En cuanto a los niveles de competitividad, el país, en el ranking global de competitividad del WEF, se ubica al año 2019, en el puesto 65; de un total de 141 naciones (World Economic Forum, 2019). Todo lo cual, configura pues una situacion de retraso innovativo-tecnológico-científico y se manifiesta en los limitados niveles de desarrollo económico y social del pais.

\subsection{RESULTADOS}

Uno de los sectores sociales que menos atención ha recibido, por parte de las políticas públicas desarrolladas por el estado peruano, es el sector educativo. Este descuido es más evidente cuando se analizan las políticas públicas universitarias, las mismas que se manifiestan en los porcentajes presupuestales asignados a las universidades públicas con relación al PBI del país. Pero este abandono es más saltante, cuando se observa la aplicación de políticas públicas específicas, destinadas al impulso de la investigación cientifica-tecnologica en las universidades, pues estas nunca constituyeron uno de los ejes centrales de las políticas de Estado a través del tiempo, con la excepción de algunas políticas públicas desarrolladas en los últimos años en este campo.

De esta manera, se ha comprobado que el Estado peruano, es uno de los que menos gasta en el impulso a la Investigación y Desarrollo $(I+D)$ en la región latinoamericana. Pues, según los 
propios organismos estatales, como el CONCYTEC, los montos asignados para estas actividades, en el año 2016, era tan solo del 0.08\% del total de su PBI, comparado con países como Colombia, Chile, y México, quienes asignan montos superiores al caso peruano. Todo lo cual confirma la cuasi inexistencia de políticas públicas de desarrollo de la ciencia y tecnología en el país.

Una de las consecuencias del descuido del Estado, a la investigación científica en el país, es que, en las universidades públicas, se ha generado un estado de inacción y retraso en el desarrolo de la ciencia y tecnología. Así lo demuestran los resultados de estas actividades, puesto que las universidades públicas muestran una escasa participación en la producción científica, tanto en el contexto de las publicaciones indexadas nacionales como las internacionales; así como una exigua producción de patentes, y un número reducido de profesores dedicados a la investigación.

La práctica de las universidades privadas, en el campo de la investigación científica, tampoco dista mucho de la realidad de las universidades públicas. Si bien, las primeras, muestran mejores índices de producción científica comparadas con estas últimas. No obstante, estas están lejos igualmente de nivelar los estándares internacionales de las universidades de la región latinoamericana. Asimismo, al haberse producido, el fenómeno de "mercantilización" (ausencia de calidad) de la educación en este tipo de universidades, por privilegiar estas ultimas la formación profesional, antes que la investigación científica, la producción de estas tampoco es significativa en el contexto universitario nacional e internacional. Con la excepción de algunas reconocidas universidades privadas.

Por su parte, la empresa privada, tampoco posee políticas de desarrollo en el campo científico tecnológico, pues no se conoce que estas tengan programas de investigación determinados, ni tampoco destinen algún presupuesto para estos fines. Ni menos promueven la generación de patentes. Lo que significa que las empresas, en particular las grandes corporaciones del país (las pequeñas y medianas, no tienen capacidad económica y financiera para hacerlo), no generan ningún tipo de know-how propio, lo que las hace importadoras netas de tecnología, de maquinaria y equipo extranjeros.

De esta forma, de una parte, las fuertes limitaciones en el campo de la investigación científica, dada las pocas posibilidades de las universidades, de generar conocimiento nuevo e innovador, $\mathrm{y}$, de otra parte, la empresa privada, que tampoco muestra interés por crear y promover este tipo de conocimiento, ha confluido en un divorcio entre ambas instituciones fundamentales del país. Así, esta separación, se revela en la ausencia de vínculos, para el desarrollo conjunto de planes y programas de investigación científica, tal como el desarrollo de proyectos tecnológicos articulados, la creación de redes de asesoramiento y apoyo tecnológico, la promoción de incubadoras tecnológicas, parques científicos y tecnológicos, y la cesión o explotación de patentes, que beneficien a la propia empresa privada, a las universidades y al desarrollo del país.

Todo lo cual, implica que el Perú, muestre niveles mínimos, en el desarrollo de la ciencia y tecnología, repercutiendo, por lo tanto, en el desarrollo de la actividad empresarial, manifestán- 
dose a su vez en la limitada diversificación productiva y la reducida generación de producción con un mayor valor agregado. Lo que significa, asimismo, que el país, como sostenemos precedentemente, se ubique en lugares poco expectantes en el ranking de innovación global, pues al año 2019, este se sitúa en el puesto 69 de un total de 129 naciones. Igualmente, en el ranking global de competitividad, en el mismo año, el país se ubica en el puesto 65, entre un total de 141 naciones. Evidenciando, una vez más, el disloque entre la academia y la empresa privada, en fines y objetivos comunes para el impulso de la ciencia y tecnología en el país.

Finalmente, es importante destacar que, en los últimos años, en particular desde el 2016, el Estado, viene impulsando una Política Nacional para el Desarrollo de la Ciencia, Tecnología e Innovación Tecnológica (CTI); tal como por ejemplo, los Proyectos de Aceleración de la Innovación, con recursos del CONCYTEC. Por su parte, algunas universidades tales como la Cayetano Heredia, Pontificia Universidad Católica del Perú, Ingeniería y la Universidad Nacional Mayor de San Marcos, desde el año 2014 vienen promoviendo la creación de 10 proyectos de parques tecnológicos, en las ciudades de Arequipa, Piura, Trujillo, Huancayo, Huánuco, Tacna y 4 en Lima (Medina, 2018).

Sin embargo, consideramos por nuestra parte, que estas iniciativas del Estado, son aún bastante insuficientes, y están lejos de constituirse en políticas públicas sostenibles en el tiempo. El ejemplo claro de este tipo de comportamiento por parte del Estado, es el monto del presupuesto público para el año 2021, destinado al desarrollo de la ciencia e innovación en el sector de la educación nacional, pues este retrocedió de \$ 2 millones de dólares a solo \$ 1 millón 600 mil dólares (Diario La República, 2020). Igualmente, el presupuesto destinado al Programa Innóvate Perú, al año 2020, fue disminuido a \$ 24 millones de dólares, de una asignación inicial el año 2015 de $\$ 32$ millones (Diario El Comercio, 2020).

\subsection{CONCLUSIONES}

La conclusión general, en función de la hipótesis también general planteado en nuestro trabajo de investigación, es que se ha demostrado que la investigación universitaria no se relaciona favorablemente con en el desarrollo empresarial en el Perú; porque la primera no desarrolla actividades de investigación científica relevante, $y$ la segunda, no se interesa por la universidad, porque esta última tiene poco que ofrecer, en términos de desarrollo científico-tecnológico. Y la empresa por su lado, no necesitaría de innovaciones, y know how, para impulsar sus actividades productivas, puesto que gran parte de estos factores para su proceso productivo los importa del extranjero. Lo que significa que existe un extendido alejamiento, entre ambas instituciones del país; cuya consecuencia no es otra que un impacto negativo en el desarrollo económico y social de la nación. 
Varios son los actores comprometidos, en el desarrollo de este escenario, en primer lugar, el Estado, por la ausencia de políticas públicas de sostén a la investigación científica, así como la insuficiencia de recursos, que este destina para impulsar la innovación científico-tecnológica en las universidades y en el país en general. Luego, las universidades, porque estas han priorizado en su actividad académica la parte formativa, descuidando la parte de la investigación científica. Y finalmente el sector empresarial, por la ausencia de planes y programas de investigación tecnológica en su seno (que propicie el desarrollo de vínculos con la universidad) y a su vez promueva la innovación y cambio tecnológico en la economía nacional.

Por consecuencia, es imperativa, la puesta en marcha y la ejecución de proyectos y programas de investigación científica conjuntos, sistemáticos, metódicos, formales y regulados; todo esto, a través del desarrollo de alianzas estratégicas entre la academia y la empresa, en un contexto de un fuerte soporte del Estado; que a la postre, esta asociación pueda convertirse en el motor del desarrollo económico y social del país. "Es fundamental mantener ese esfuerzo de recomponer la gobernanza y la institucionalidad de las actividades de ciencia, tecnología e innovación en el país, ya que sin ciencia y tecnología no hay futuro", escribe el (Diario El Comercio, 2020) citando al presidente de la Republica Francisco Sagasti, en su discurso de asunción del mando.

\section{REFERENCIAS BIBLIOGRÁFICAS}

(GII), G. I. (2019). Informe 2019. Obtenido de: https://www.translatetheweb.com/?from =en\&to=es\&ref=SERP\&refd=www.bing.com\&dl=es\&rr=UC\&a=https\%3a\%2f\%2fwww. globalinnovationindex.org\%2fgii-2019-report

(WEF), World Economic Forum. (2019). The Global Competitiveness Report 2019. Obtenido de: http://www3.weforum.org/docs/WEF_TheGlobalCompetitivenessReport2019.pdf

Andina. (2011). Obtenido de:

https://www.educacionenred.pe/noticia/?portada=9353\#ixzz6Pjqha8tO

Banco Central de Reserva del Perú (BCRP). (2019). Memoria 2018. Obtenido de: https://www.bcrp.gob.pe/publicaciones/memoria-anual/memoria-2018.html

Banco Mundial. (2019). Gasto público en educación. Obtenido de: https://datos.bancomundial.org/indicador/SE.XPD.TOTL.GD.ZS 
BBVA Research. (2014). Competitividad del Sector Mamufacturero en America Latina: Tendencias y Determinantes. Documento de trabajo. Obtenido de: https://www.bbvaresearch. com/wp-content/uploads/mult/WP_1410_tcm346-430744.pdf

Boiral, O. \&. (Diciembre de 2016). La investigación en dirección y gestión de empresas: ¿una actividad relevante y fundamentada en la realidad? Revista de Dirección y Administración de Empresas(23), 1-12. Obtenido de: https://addi.ehu.es/handle/10810/20328

Cabezas, C. (2006). Rol del Estado en la investigación científica en salud y transparencia en la información. 23(4). Obtenido de:

http://www.scielo.org.pe/scielo.php?pid=S1726-46342006000400008\&script=sci_abstract

Chuquispuma, E. (2016). Estrategias para el Desarrollo de una Cultura en Patentes en las Tres Universidades del Perú con Mayor Cantidad de Publicaciones Científicas. Universidad Peruana Cayetano Heredia. Obtenido de: http://repositorio.concytec.gob.pe/ bitstream/20.500.12390/321/3/2016_Chuquispuma_Estrategias-para-el-desarrollo-deuna-cultura-en-patentes.pdf

Comisión Nacional por la Segunda Reforma Universitaria.(2002). Diagnóstico dela Universidad Peruana: Razones para una nueva reforma universitaria. Obtenido de: http://www4.congreso. gob.pe/congresista/2001/liberico/universidad-final.pdf

CONCYTEC. (2016). Política nacional para el desarrollo de la ciencia, tecnología, e innovación tecnológica-CTI. Obtenido de:

https://portal.concytec.gob.pe/images/documentos/Politica_Nacional_CTI-2016.pdf

CONCYTEC. (2017). I Censo nacional de investigación y desarrollo de centros de investigación. Obtenido de: https://www.gob.pe/concytec

CONCYTEC. (2017). Primer censo revela baja inversión en investigación y desarrollo en el Perú. Lima, Perú. Obtenido de: https://portal.concytec.gob.pe/index.php/noticias/1051primer-censo-revela-baja-inversion-en-investigacion-y-desarrollo-en-el-peru

CONCYTEC. (2018). Concytec pone en marcha proyecto con el Banco Mundial. Obtenido de: https://portal.concytec.gob.pe/index.php/noticias/1284-concytec-pone-en-marcha-proyectocon-el-banco-mundial-de-100-millones-de-dolares-con-enfasis-en-seis-sectores-economicos

CONCYTEC. (2020). ¿Quienes somos? Obtenido de: https://portal.concytec.gob.pe/ 
Diario El Comercio. (19 de Noviembre de 2020). Especialistas piden mejorar la gobernanza para ciencia y tecnología en lo que resta del gobierno. Obtenido de: https://elcomercio.pe/ economia/peru/especialistas-piden-mejorar-la-gobernanza-para-ciencia-y-tecnologia-en-loque-resta-del-gobierno-ncze-noticia/

Diario La Republica. (9 de Septiembre de 2020). Se destinarán S/ 1,5 millones menos para ciencia e innovación. Obtenido de: https://larepublica.pe/economia/2020/09/01/presupuestose-destinaran-15-millones-de-soles-menos-para-ciencia-e-innovacion/

Figrup. (2018). ¿Cuáles son las empresas que más invierten en $I+D$ del mundo? Obtenido de: https://www.f-iniciativas.es/\%C2\%BFcu\%C3\%A1les-son-las-empresas-que-m\%C3\%A1sinvierten-en-id-del-mundo

Figueroa,V.M.(2013).Elroldela universidadeneldesarrollo. La perspectiva de los organismos internacionales. Obtenido de: Academia.edu https://www.academia.edu/7355604/El_papel_ de_la_universidad_en_el_desarrollo

FONDECYT. (2019). Proyectos de aceleración de la innovación. Obtenido de: https://fondecyt. gob.pe/convocatorias/innovacion-y-transferencia-tecnologica/aceleracion-de-la-innovacion

Gestión. (5 de 11 de 2019). Entre los países de la región, Perú cuenta con la mayor cantidad de emprendedoras. Obtenido de: https://gestion.pe/economia/empresas/entre-los-paises-de-laregion-peru-cuenta-con-la-mayor-cantidad-de-emprendedoras-noticia/?ref=gesr

Global Innovation Index. (2019). Informe 2019. Obtenido de: https://www.translatetheweb. $\mathrm{com} /$ ?from=en\&to=es\&ref=SERP\&refd=www.bing.com\&dl=es\&rr=UC\&a=https\%3a\%2f\%2fw ww.globalinnovationindex.org\%2fgii-2019-report

Hidalgo, M. .. (2015). Investigación científica en la universidad pública peruana y su relación con el estado y empresa. (F. d. UNMSM, Ed.) Quipukamayoc, 23(44), 95-101.

INEI. (2016). Estructura Empresarial Según Actividades Económicas. Obtenido de: https://www.inei.gob.pe/media/MenuRecursivo/publicaciones_digitales/Est/Lib1445/capo2.pdf

INEI. (2018). INEI ejecuta la Encuesta Nacional de Empresas 2018. Lima, Perú. Obtenido de: http://m.inei.gob.pe/prensa/noticias/inei-ejecuta-la-encuesta-nacional-de-empresas-2018-11552/ 
InnovatePeru. (6 de Octubre de 2020). Innovate Perú. Recuperado el 6 de Octubre de 2020, de: https://www.innovateperu.gob.pe/quienes-somos/innova-como-ellos

Ismodes, E. (2015). Obstáculos a la vinculación universidad empresa en el Perú: ¿Qué hacer? Lima, Perú. Obtenido de:

https://www.pucp.edu.pe/profesor/anibal-ismodes-cascon/publicaciones/?x\&pagina=1

Kantis, H. (2020). Los emprendimientos de base científico-tecnológica en América Latina. BID. Obtenido de: https://www.virtualpro.co/noticias/-y-los-emprendimientos-cientificotecnologicos-en-america-latina-

Larraín, F. y Sachs, J. (2004). Macroeconomia en la economia global. Prentice Hall y Pearson Educacion SA.

MCPRO. (2018). Amazon es la empresa que más invierte en investigación y desarrollo en Estados Unidos. Obtenido de:

https://www.muycomputerpro.com/2018/04/10/amazon-investigacion-desarrollo-estados-unidos

Medina, D. (2018). El rol de las universidades peruanas frente a la investigacion y el desarollo tecnologico. Propósitos y Representaciones, 6(2), 703-737.

doi: http:/dx.doi.org/10.20511/pyr2018.v6n2.244

Ministerio de la Producción del Perú. (2015). Estudio de la Situación Actual de las empresas peruanas. Obtenido de:

https://cdn.www.gob.pe/uploads/document/file/299953/d29294_opt.pdf

Palmieri, F. (2017). Informe PuntoEdu sobre la situación de las universidades públicas en el Perú. Pontificia Universidad Católica del Perú. Obtenido de: https://puntoedu.pucp.edu. pe/noticias/informe-puntoedu-sobre-la-situacion-de-las-universidades-publicas-en-el-peru/

Perú21. (2020).Presupuesto 2020. Obtenidode: https://peru21.pe/economia/presupuesto-2020destinaran-s-4-588-millones-universidades-publicas-informo-solar-500090-noticia/?ref=p21r

PRONABEC. (2020). Portal de transferencia. Obtenido de: https://www.pronabec.gob.pe/beca-presidente/ 
José Adolfo Hinojosa Pérez | Fernando Polo Orellana

$132<\quad$ La investigación científica universitariay su relación con el desarrollo empresarial en el Perú Págs. 117 -133. DOI: http://dx.doi.org/10.30972/rfce.2725661

Pulido, V. (2019). La situacion de la investigacion y su desarrollo en el Peru: Reflejo de estado actual de la universidad peruana. Pensamiento \& Gestion(46).

doi: https://doi.org/10.14482/pege.46.7615

Rodríguez, M. A. (2015). Vinculación Universidad - Empresa - Estado en Nicaragüa. Revista Científica Electrónica de Ciencias Humanas, 11(31), 15-34. Obtenido de: https://search.proquest.com/docview/1912188304/5E250582435C4F1DPQ/5?accountid=14747

Sánchez, J. Z. (2016). Investigación científica y responsabilidad social: factores de impacto en las instituciones de educación superior del Ecuador. Cofin, 11(2), 200-213. Obtenido de: http://scielo.sld.cu/scielo.php?script=sci_arttext\&pid=S2073-60612016000200011

Sarabia, G. (Junio de 2016). La vinculación universidad-empresa y sus canales de interacción desde la perspectiva de la academia, de la empresa y de las. Ciencia UAT, 1O(2). Obtenido de: http://www.scielo.org.mx/scielo.php?script=sci_arttext\&pid=\$2007-78582016000100013

SUNEDU. (2019). Universidades. Obtenido de: https://www.sunedu.gob.pe/lista-universidades/

Thomson Reuters. (2018). The Top 100 Global. Obtenido de: https://www.thomsonreuters. com/content/dam/ewp-m/documents/thomsonreuters/en/pdf/reports/thomson-reuters-top100-global-tech-leaders-report.pdf

Vega, J. M. (Septiembre de 2011). Las relaciones universidad-empresa: tendencias y desafíos en el marco del Espacio Iberoamericano del Conocimiento. Revista Iberoamericana de Eduación(57), 109-124. Obtenido de: rieoei.org/RIE/article/view/488

World Economic Forum. (2019). The Global Competitiveness Report 2019. Obtenido de: http://www3.weforum.org/docs/WEF_TheGlobalCompetitivenessReport2019.pdf 


\section{CURRICULUM VITAE}

\section{José Adolfo Hinojosa Pérez}

Economista. Doctor en Ciencias Económicas por la Universidad de Paris-Francia. Miembro del Comité de Calidad de la Unidad de Postgrado de la Facultad de Ciencias Económicas de la Universidad Nacional Mayor de San Marcos, Lima-Perú.

jhinojosap@unmsm.edu.pe

\section{Fernando Polo Orellana}

Contador Público. Magister en Tributación y Política Fiscal. Candidato a doctor por la Universidad de San Martín de Porres, Lima-` Perú.

d.fpolo@upla.edu.pe 